\title{
Sinatra, He3nry und andere moderne Enzyklopädisten. Synchron und diachron vergleichende Anmerkungen zur Eigen- und Fremdbenennung von Wikipedia-Autoren*
}

\author{
Joachim Grzega (Eichstätt)
}

\begin{abstract}
This paper analyzes the formation of authors' pseudonyms in the English, German, French, Italian and Spanish versions of the Internet encyclopedia Wikipedia. 694 semi-randomly collected Wikipedia usernames ("nicknames") are checked against traditional classifications of pseudonyms and compared to a semi-randomly collected corpus of 607 pseudonyms of English, German, French, Italian and Spanish writers of the $18^{\text {th }}$ and $19^{\text {th }}$ centuries (who published, at least in part, non-fictional works). The main results of the diachronic comparison are the following: (1) Qualitatively, typical modern phenomena are the replacement of letters by similar-looking special characters (called leetspeak, e. g. Serg!o for Sergio), the use of abstract nouns, the use of non-standard forms, deacronymization (e.g. when a Spanish Wikipedian's initials $F G$ become Efegé), and the formation of nicknames from Wikipedia pseudonyms on users' discussion pages ("nick-nicknames"). (2) Qualitatively, typical historical phenomena are the use of symbols not easily representable on modern (Latin) keyboards (e. g. musical notes and Greek letters). (3) Quantitatively, there is a clear decrease in the use of Latin elements in pseudonyms. A cross-linguistic comparison of Wikipedia nicknames leads to the following characteristic features of the different Wikipedia communities: (a) English Wikipedia nicknames show the highest amount of non-onymic elements and numbers and the lowest amount of nicknames with foreign elements. (b) French-speaking Wikipedians use the lowest amount of numbers in their nicknames and are the least informative as regards telling the motivation behind their usernames. (c) Italian-speaking Wikipedians are the most open to tell about the motivation behind their usernames, use relatively few non-onymic elements in their nicknames, but are very productive as to forming nicknicknames. (d) Spanish-speaking Wikipedians use relatively few appellative lexemes, are the most reserved as to using foreign elements, but use clippings relatively often and are very productive as to forming nicknicknames. (e) German-speaking Wikipedians are most open to using foreign elements.
\end{abstract}

\section{$1 \quad$ Vorbemerkungen}

Einer der Autoren der französischen Encyclopédie (oder Dictionnaire raisonné des sciences, des arts et des métiers) im 18. Jh. schrieb in seinem Leben unter 174 verschiedenen Ersatznamen. Sein eigentlicher Name war François Marie Arouet le Jeune; wohl der berühmteste seiner Ersatznamen war Voltaire, ein Anagramm aus seinem Nachnamen und der Abkürzung L. J. (Le Jeune). Der englische Essayist und Schriftsteller mit dem amtlichen Namen Daniel

\footnotetext{
* Ich danke Bea Klüsener, Ursula Lenker sowie zwei anonymen Gutachtern für wertvolle Hinweise zu einer früheren Version dieses Aufsatzes.
} 
Foe verwendete sogar rund 200 Namen, von denen der berühmteste Daniel Defoe war - eine Selbstadelung seines Namens als Pseudonym (vgl. dazu die Auflistungen in Room 1981: 332-337).

Wie sieht die Verwendung von Pseudonymen im Zeitalter der Internet-Enzyklopädie Wikipedia aus? Eine Frage, der bislang in der Literatur offenbar noch nicht nachgegangen worden ist. Welche Typen von Pseudonymen verwenden Wikipedia-Autoren? Welche Typen scheinen besonders häufig? Welche Unterschiede gibt es zwischen englischen, französischen, italienischen, spanischen und deutschen Wikipedia-Pseudonymen? Das sind die Fragen dieses Beitrages.

Zunächst ist zu klären, was unter einem Pseudonym zu verstehen ist. Ratier (1993-1994) übernimmt in seiner Encyclopédie des pseudonymes eine Einteilung von Littré und unterscheidet zwischen (1) Pseudonym ("lügnerischer Name"), (2) Kryptonym (Anagramm), (3) Heteronym (Name einer anderen Person). Ein Heteronym sei dabei abzugrenzen vom Phänomen des "Ghostwriters" (So gebe die Encyclopedia Britannica als Autor des Artikels "Mass Production" Henry Ford an, vermutlich stecke aber sein persönlicher Publizist als Ghostwriter dahinter, ebenso wie der Faschismus-Artikel der Enciclopedia italiana nicht von Benito Mussolini selbst, sondern vom italienischen Philosophen Giovanni Gentile stamme). Andere Autoren verstehen Pseudonym in einem weiteren Sinne, der alle drei Unterphänomene bei Ratier zusammenfasst. Die Definition lato sensu soll auch diesem Beitrag zu Grunde gelegt werden.

Nun stellt sich die Frage, warum man sich überhaupt ein Pseudonym zulegen soll. Adrian Room (1981: 15-22) nennt eine Reihe von Gründen, die jemanden zur Annahme eines verschleiernden Pseudonyms bewegen können, unter anderem

- Demonstration gesellschaftlicher Integration

- Demonstration unterschiedlicher Lebensbereiche

- unpassender/unschöner echter Name

- Vermeidung des Gleichklangs mit dem Namen einer anderen Person

- Schaffung eines Gleichklangs mit dem Namen einer anderen Person

- Verschleierung seines Geschlechts.

Bei Autoren sei es vor allem das Aufsetzen einer literarischen Maske, möglicherweise um Ruhm zu entfliehen ("flight from fame") oder um einer Zensur oder gar Repressalien zu entgehen - selbst wenn für die klassische enzyklopädische Mitarbeit neue Pseudonyme heute nicht mehr zu erwarten seien; zur Mitarbeit gebeten habe man die Personen ja aufgrund ihres guten Namens. Dieser natürlich konnte real oder ursprünglich verschleiernd sein.

Albert Dauzat (1942: 180) präzisiert, dass solche Gründe wie die genannten erst seit der Renaissance gälten. Im Mittelalter, wo sich die Rufnamen aus einer geringen Zahl bevorzugter Namen speisten und feste Familiennamen noch fehlten oder selten waren (feste Familiennamen gab es in Europa erst allmählich ab dem Hochmittelalter), dienten Namensänderungen oder Namenszusätze nicht der Verschleierung, sondern der besseren Identifizierung eines Autors. In der Wikipedia finden wir nun beides: das Angebot zur Verschleierung und den Zwang zur eindeutigen Identifizierbarkeit. Letzteres allerdings verbindet sich auch wieder mit einem Angebot, etwas über sich zu verraten.

\section{$2 \quad$ Namensrecht in den Wikipedia-Gemeinden}

Was bedeutet Zwang zur eindeutigen Identifizierung? Sofern ein Wikipedia-Autor nicht einfach unter der eindeutigen Adresse seines Computeranschlusses etwas beitragen will, muss er sich einen Benutzernamen, einen so genannten Nickname zulegen. Nickname ist eigentlich irreführend, da traditionelle Nicknames, also 'Spitznamen' typischerweise nicht autonom, 
sondern heteronom gebildet werden. Der Begriff hat sich jedoch in allen fünf untersuchten Sprachen als Terminus für 'Internet-Benutzername' eingebürgert. Dieser Benutzername bleibt dann innerhalb der Gemeinde einer Wikipedia-Sprachversion einmalig. Unter den WikipediaAutoren kann es also nie zu Heteronymen im engeren Sinne kommen, d. h., WikipediaAutoren des gleichen Namens gibt es nicht. Wenn man sich als Max Mustermann anmelden will, aber ein anderer Max Mustermann dies bereits getan hat, muss man einen anderen Namen wählen - und sei der Unterschied auch noch so gering. So lesen wir auf der Benutzerseite des deutschen Wikipedianers He3nry Folgendes:

Als Bewunderer von Tom Lehrer verwende ich eine englische Variante meines Namens, die in einer Anmoderation zu einem seiner besten Songs vorkommt. Da englischsprachige Menschen mir den Namen aber immer schon weggeschnappt haben, verschiebe ich die "3": "I am reminded at this point of a fellow I used to know whose name was Henry, only to give you an idea of what a individualist he was, he spelled it H-E-N-3-R-Y, the three was silent, you see."

Innerhalb einer Sprachversion der Wikipedia können nicht zwei Personen den gleichen Benutzernamen tragen. Wohl kann es aber sein, dass der Benutzer mit dem Benutzernamen Max Mustermann der deutschsprachigen Wikipedia nicht die gleiche Person ist wie der Max Mustermann der englischen Wikipedia, So sind etwa der Sinatra der deutschen Wikipedia, jener der englischen und jener der französischen Wikipedia ein und dieselbe Person. Dagegen verbergen sich hinter dem Sinatra der spanischen Wikipedia und dem Sinatra der italienischen Wikipedia andere Personen.

Man kann sich auch mehrfach registrieren und somit mehrere Pseudonyme haben. Solche Mehrfach-Identitäten bezeichnet man als Sockpuppets, und wenn man diese MehrfachIdentitäten auf den entsprechenden Benutzerseiten nicht eindeutig bekannt macht, gilt das innerhalb der Wikipedia als unethisch (vgl. http://de.wikipedia.org/wiki/Wikipedia:Sockenpuppe und die von dort aus zugänglichen äquivalenten Seiten in anderen Sprachversionen). Man macht sich mit Sockpuppets schnell verdächtig, Vandalismus betreiben und entsprechende Sanktionen umgehen zu wollen (und dies wird von Wikipedia-Administratoren auf Grund der Arten und Orte von Beiträgern auch aufgedeckt). Anders verhält es sich dagegen bei offenen Mehrfachidentitäten, etwa bei folgendem französischen Wikipedianer, der auf seiner Wikipedia-Benutzerseite schreibt:

En date du 9 décembre 2008, le compte Sherbrooke ne me servira presque plus. J'ai décidé de devenir Cantons-de-l'Est, hommage à la région où j'habite. ['Mit Datum 9. Dezember 2008 werde ich das Benutzerkonto Sherbrooke kaum mehr benutzen. Ich habe beschlossen Cantonsde-l'Est zu werden, als Hommage an die Region, in der ich wohne.']

Wikipedia-Nicknames werden also durchaus mit viel Bedacht gewählt.

\section{$3 \quad$ Korpuserstellung}

Zur Erstellung eines Korpus sind folgende Elemente der Wikipedia genutzt worden. Zu jedem Artikel in der Wikipedia gibt es nicht nur eine Diskussions-Seite, sondern auch eine Seite "history" bzw. "historique" bzw. "cronologia" bzw. "historial" bzw. "Versionen/Autoren". Die deutsche Bezeichnung spricht für sich. Hier kann man nachlesen, wer jeweils wann welche Änderung an einem Beitrag vorgenommen hat. Die englische Wikipedia-Eintrag für "Europe" (Grafik 1) und die dazugehörige Versionenseite (Grafik 2) seien hier als Beispiel gezeigt. 


ja, the free encyclopedia - Mozilla Firefox
sicht Chronik Lesezeichen Extras Hilfe
Kostenlose Hotmail $\mathbf{W}$ Links anpassen [D Windows Media $\square$ Windows
Heip us provide free content to the worid by donating today!
article discussion wiew source history
Europe

From Wikipedia, the free encyclopedia

For other uses, see Europe (disambiguation).

Europe (pronounced/jarap/ or /juarap/) is, by convention, one of the world's seven continents. Comprising the westernmost peninsula of Eurasia, Europe is generally divided from Asia to its east by the water divide of the Ural Mountains, the Ural River, the Caspian Sea, and by the Caucasus Mountains, the Kuma-Manych Depression, and the Black Sea to the southeast. ${ }^{[1]}$ Europe is washed upon to the north by the Arctic Ocean and other bodies of water, to the west by the Atlantic Ocean, to the south by the Mediterranean Sea, and to the southeast by the Black Sea and the waterways connecting it to the Mediterranean. Yet the borders for Europe-a concept dating back to classical antiquity-are somewhat arbitrary, as the term continent can refer to a cultural and political distinction or a physiographic one. Malta was considered an island of Africa for centuries. ${ }^{[2]}$

Europe is the world's second-smallest continent by surface area, covering about 10,180,000 square kilometres $(3,930,000 \mathrm{sq} \mathrm{mi)}$ or $2 \%$ of the Earth's surface and about $6.8 \%$ of its land area. Of Europe's approximately 50 states, Russia is the largest by both area and population, while the Vatican City is the smallest. Europe is the third most populous continent after Asia and Africa, with a population of 731 million or about $11 \%$ of the world's population; however, according to the United Nations (medium estimate), Europe's share may fall to about $7 \%$ in $2050 .{ }^{[3]}$ In 1900, Europe's share of the world's population was $25 \%{ }^{[4]}$

Europe, in particular Ancient Greece, is the birthplace of Western culture. ${ }^{[5]}$ It played a predominant role in global affairs from the 16th century onwards, especially atter the beginning of colonialism. Between the 16 th and 20th centuries, European nations controlled at various times the Americas, most of Africa, Oceania, and large portions of Asia. Both World Wars were ignited in Central Europe, greatly contributing to a decline in European dominance in world affairs by the mid-20th century as the United States and Soviet Union took prominence. During the Cold War Europe was divided along the lron Curtain between NATO in the West and the Warsaw Pact in the East. European integration led to the formation of the Council of Europe and the European Union in Western Europe, both of which have been expanding eastward since the fall of the Soviet Union in 1991.

\begin{tabular}{|l|}
\multicolumn{1}{|c|}{ Contents [hide] } \\
1 Definition \\
2 Etymology \\
3 History \\
3.1 Prehistory \\
3.2 Classical antiquity
\end{tabular}

\section{Grafik 1}




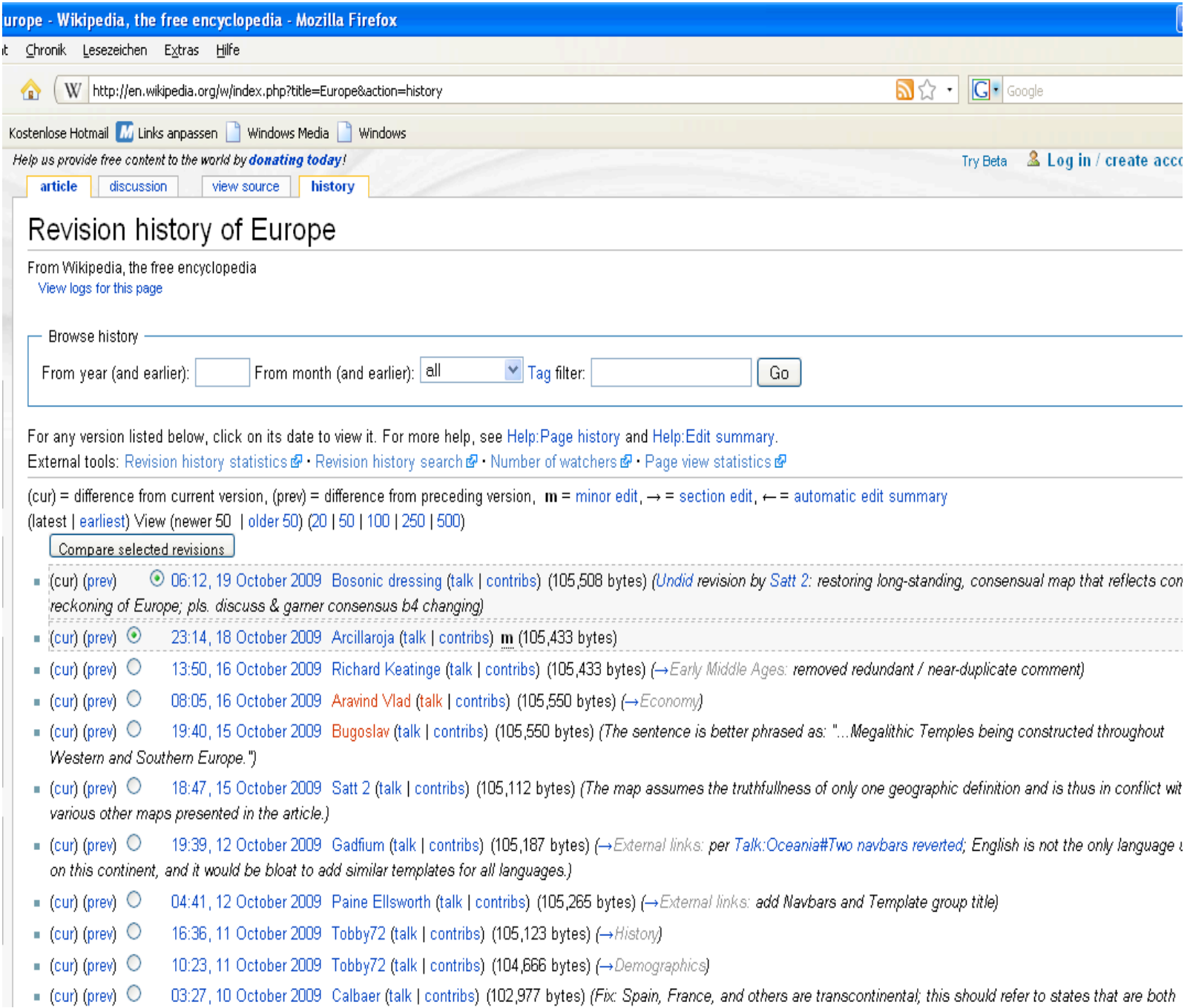

\section{Grafik 2}

$\mathrm{Zu}$ den Stichwörtern "Historische Linguistik", "Voltaire", "Europa", "Onomasiologie", "Wikipedia", "Tokio Hotel" und "James Bond" habe ich die Nicknames der mit einer Benutzerseite ausgestatteten Autoren der zum Zeitpunkt der Korpuserstellung (Oktober bis Dezember 2009) je 100 neuesten Ergänzungen in der englischen, französischen, italienischen, spanischen und deutschen Wikipedia gesammelt und deren Personenseiten gesichtet. Dabei ergab sich insgesamt eine Sammlung von 694 verschiedenen Pseudonymen von WikipediaAutoren mit Benutzerseiten. Sie verteilen sich auf die Wikipedia-Sprachgemeinschaften wie in Tabelle 1 dargestellt.

\begin{tabular}{|c|c|c|c|c|c|}
\hline gesamt & engl. & dt. & fr. & it. & sp. \\
\hline $694=100 \%$ & $193=28 \%$ & $185=27 \%$ & $139=20 \%$ & $95=14 \%$ & $82=12 \%$ \\
\hline
\end{tabular}

\section{Tabelle 1}

Der relativ geringe Anteil der italienischen und spanischen Wikipedia-Benutzernamen ist dadurch zu erklären, dass hier einerseits die Zahl der aktiven Wikipedianer noch relativ gering ist (auf den Seiten tauchen immer wieder die gleichen Autoren auf) und dass andererseits relativ viele Autoren sich keinen Benutzernamen zulegen, sondern etwas unter der InternetProtokoll-Adresse ihres Rechners abspeichern. 
Nur eine Minderheit der Autoren gibt auf ihren Benutzer-Seiten die Motivation hinter ihrem Pseudonym an, nämlich insgesamt 163 von 694 Wikipedianern, d. h. $23 \%$. Tabelle 2 präsentiert die sprachenspezifische Aufgliederung:

\begin{tabular}{|c|c|c|c|c|c|}
\hline gesamt & engl. & dt. & fr. & it. & sp. \\
\hline 163 von $694=$ & 43 von $193=$ & 51 von $185=$ & 21 von $139=$ & 29 von $95=$ & 19 von $82=$ \\
$23 \%$ & $23 \%$ & $28 \%$ & $15 \%$ & $31 \%$ & $23 \%$ \\
\hline
\end{tabular}

Tabelle 2

Am mitteilungsfreudigsten sind demnach die Autoren der italienischsprachigen Wikipedia, am wenigsten die Autoren der französischsprachigen Wikipedia. Unter den italienischen Wikipedia-Benutzernamen gibt es also den höchsten Grad an Namen, deren Benennungsmotiv bekannt ist. Trotz der insgesamt geringen Zahl an Motivationsklärungen lassen sich eine Reihe von qualitativen und einige quantitative Angaben machen und Vergleiche zu klassischen Pseudonymen von Autoren ziehen.

Zum historischen Vergleich ist ein historisches Korpus mit Hilfe von William Cushings (1886: 325-487) Werk erstellt worden, das sowohl englische als auch international berühmte nicht-englischsprachige Autoren bzw. enzyklopädisch wissensvermittelnde und wissensbildende Personen des 18. und 19. Jahrhunderts mit ihren Pseudonymen vorstellt. Unter dem Buchstaben A lassen sich 438 Pseudonyme englischer Autoren finden, unter den Buchstaben A und B 81 Pseudonyme französischer und unter den Buchstaben A-G sowie L 64 Pseudonyme deutscher Autoren, 10 Pseudonyme italienischer und 14 Pseudonyme spanischer Autoren. Dies führt zu einem Gesamtkorpus von 607 historischen Pseudonymen.

\section{Korpusanalyse 1: Anmerkungen zu den (autonomen) Nicknames}

Im HSK-Band zur Namenkunde (Eichler et al. 1995) fehlt leider eine morphosemantische Klassifikation von Pseudonymen. Für einen Vergleich sollen daher die Klassifizierungen aus der Studie von Edwin Bormann (1901, insbesonere 14-36), der ältesten umfassend systematischen Studie zu Pseudonymen, und jener von Adrian Room (1981, insbesondere 23-63), der ältesten Studie an einem internationalen Pseudonymenkorpus, dienen. Die Klassifizierungen unterscheiden zunächst grob zwischen solchen Pseudonymen, die sich formal auf den Realnamen zurückführen lassen, und solchen, die in einem sachlichen Zusammenhang mit dem Namensträger stehen. Die Klassifizierung in Untertypen beruht auf formalen Kriterien. Dabei ist zu unterstreichen, dass die einzelnen Typen in einem Pseudonym auch kombiniert sein können. In den Werken von Bormann und Room werden die einzelnen Typen nicht mit Fachtermini versehen, sondern nur beschrieben. Im Folgenden wird daher versucht, diese Beschreibungen in nominalen Schlagworten zu fassen. Einige formale Typen lassen sich mit den Typen bei Bormann und Room nicht fassen; sie werden im Folgenden ergänzend angeführt.

\subsection{Eigener Name als Basis (proprium-derivative Pseudonyme)}

\subsubsection{Xenophonierung des Namens}

Unter Xenophonierung sei der Vorgang verstanden, seinem Namen einen "fremdsprachlichen Anstrich" zu geben (dies ist nicht zu verwechseln mit einer Übersetzung des Namens, die unter Punkt 4.1.5 genannt ist). Die Xenophonierung eines Namens liegt etwa vor beim spanischen Wikipedianer Diegusjaimes, der eigentlich Diego Fernando Jaimes González heißt, und beim italienischen Wikipedianer Domyinik, der eigentlich Domenico heißt. Auch der schon erwähnte He3nry schreibt, dass er eine englische Variante seines Namens nehme. 
Ob der französische Wikipedianer Luscianusbeneditus eigentlich Lucien Benoît heißt oder sein Name anderer Motivation entspringt, verrät er nicht. Im historischen Korpus taucht der xenophonierte Typ nur 4-mal auf (also in weniger als $1 \%$ der Fälle): es sind die Pseudonyme Petrus Borel und Peter Borel für Pierre Borel d'Hauterive, das Pseudonym Robert Byr für Robert Bayer und das Pseudonym Izak für den Mittelnamen von John Isaac Adams.

\subsubsection{Indigenisierung des Namens}

Die Indigenisierung stellt das Gegenstück zur Xenophonierung dar. Hier wird einem Realnamen nicht-muttersprachlicher Herkunft ein "muttersprachlicher Anstrich" gegeben. Dieser Typ liegt bei den Wikipedia-Nicknames ein einziges Mal vor, und zwar bei der französischen Wikipedianerin Léa, die sich eigentlich Leah schreibt. Im historischen Korpus taucht dieser Typ streng genommen nicht auf (lediglich in Amalie Heiter könnte man im Vornamen eine Indigenisierung von Amalia, Herzogin von Sachsen sehen, allerdings ist [-iə] kein deutscher Wortauslaut).

\subsubsection{Verwendung eines Kosenamens}

Kosenamen, die vom Vornamen abgeleitet sind und nicht nur Kürzungen darstellen (die unter dem Punkt 4.1.7., Clippings, aufgelistet sind), sind unter den Wikipedia-Nicknames wenig präsent: sie liegen vor bei dem spanischen Wikipedianer Javierito92 und bei dem italienischen Wikipedianer Vituzzu, der eigentlich Vito heißt. Unter den deutsch-, englisch- und französischsprachigen Wikipedianern taucht der Typ nicht auf. Im historischen Korpus kann man eventuell folgenden Fall dazurechnen, der allerdings nur in Kombination mit einem anderen Typ auftritt: bei Kate De Courcy statt Katherine Armstrong. Ob in Wattie Rushton ein Hypochoristikon zum Vornamen in A. Watson Atwood stecken soll, ist unklar. Ansonsten tauchen Kosenamen nur als Kürzungen auf, allerdings auch dies nur bei den Franzosen Sam und Léon für Samuel Henri Berthroud und Léonard Boitel.

\subsubsection{Synonymisierung des Namens}

Unter Synonymisierung sei der Vorgang verstanden, die nicht-onymischen Elemente in einem Namen (wie sie auch in der Sprache des Namensträgers vorkommen können) durch synonyme Morpheme auszutauschen. $\mathrm{Zu}$ diesem Typ gibt es kein erkennbares Beispiel in unserem Korpus; der Typ ist aber durchaus möglich. Auch historisch taucht diese Möglichkeit verhältnismäßig selten auf - in unserem Korpus gerade ein einziges Mal (George Archers Pseudonym Hesper Bendbow, dessen zweiter Bestandteil Archer synonymisiert).

\subsection{5 Übersetzung des Namens}

Unter Übersetzung eines Namens sei der Vorgang verstanden, die nicht-onymischen Elemente in einem Namen (wie sie auch in der Sprache des Namensträgers vorkommen können) durch Morpheme zu übersetzen. Zu diesem Typ gibt es weder im Wikipedia-Korpus noch im historischen Korpus ein erkennbares Beispiel, außer man berücksichtigt Laicus als eine Übersetzung des volksetymologisch zu Layman gestellten Vornamens in Lyman Abbott.

\subsubsection{Silbenspiel}

Unter Silbenspiel sei das Verschieben von Silben verstanden. Dies ist ein dritter Typ, zu dem es kein erkennbares Beispiel in unserem Korpus gibt; der Typ ist freilich möglich. Auch historisch taucht diese Möglichkeit verhältnismäßig selten auf. In unserem historischen Korpus findet sich das Silbenspiel 8-mal (also in gut $1 \%$ der Fälle). Beispiele sind das Pseudonym Bernd von Guseck eines deutschen Mathematikers und Romanciers namens 
Gustav von Berneck und das Pseudonym Adolf Brennglas eines deutschen Satirikers namens Adolf Glaßbrenner).

\subsubsection{Clipping/Wortkürzung}

Unter Clipping sei entsprechend einer allgemein linguistischen Definition das Abschneiden von nicht-morphematischen Bestandteilen verstanden. Clipping zeigt sich bei den spanischen Wikipedianern Almend, eigentlich Luis Alberto Mendieta Acosta, Sanbec, eigentlich Santiago Becerra Carrillo, und Mel_23, eigentlich Melisa. Beim italienischen Wikipedianer Maso wird an den übrig gebliebenen Bestandteil von Lorenzo Masetti noch ein $o$ gehängt, damit die phonotaktischen Regeln respektiert werden. Bei den spanischen Clippings hingegen fällt auf, dass viele die phonotaktischen Regeln verletzen. Damit verhalten sich onymische Clippings anders als appellative Clippings. So werden etwa bicicleta und facultad zu bici und facu gekürzt, nicht zu *bic oder *fac (vergleiche dagegen das Pseudonym Sanbec). Überhaupt tauchen sichtbare Clippings in der spanischen Version prozentual deutlich häufiger auf als in den anderen Wikipedia-Versionen: Tabelle 3 zeigt den Anteil gemessen an der Gesamtzahl der gesammelten Nicknames pro Sprachversion, Tabelle 4 zeigt den Anteil gemessen an der Zahl der Nicknames, bei denen das Benennungsmotiv bekannt ist.

\begin{tabular}{|c|c|c|c|c|c|}
\hline gesamt & engl. & dt. & fr. & it. & sp. \\
\hline 30 von $694=$ & 7 von $193=$ & 3 von $185=$ & 1 von $139=$ & 5 von $95=$ & 14 von $82=$ \\
$5 \%$ & $4 \%$ & $2 \%$ & $0 \%$ & $5 \%$ & $17 \%$ \\
\hline
\end{tabular}

Tabelle 3

\begin{tabular}{|c|c|c|c|c|c|}
\hline gesamt & engl. & dt. & fr. & it. & sp. \\
\hline 30 von $694=$ & 7 von $43=$ & 3 von $51=$ & 1 von $21=$ & 5 von $29=$ & 14 von $19=$ \\
$5 \%$ & $16 \%$ & $6 \%$ & $5 \%$ & $17 \%$ & $74 \%$ \\
\hline
\end{tabular}

Tabelle 4

Im historischen Korpus taucht der Fall nur 4-mal auf (also in weniger als $1 \%$ der Fälle), davon 2-mal beim Italiener mit dem Realnamen Arnoldo Fusinato, der sowohl unter Don Fuso als auch unter Don Fusina schreibt.

\subsubsection{Initialbildung}

In unserem Korpus kommt die Bildung eines Pseudonyms mit Hilfe der Initialen des Realnamens nur in $4 \%$ der Pseudonyme mit bekanntem Benennungsmotiv vor, selbst wenn wir Kombinationen mit Zahlen einrechnen (d. h. je 1-mal unter den englischen, italienischen und spanischen und je 2-mal unter den deutschen und französischen Namen). Bei der Analyse des historischen Korpus fällt auf, dass der Gebrauch von Initialien der zweithäufigste Typ ist. Unter den 438 Pseudonymen der englischsprachigen Autoren sind 75 nach diesem Typ gebildet $(17 \%)$, unter den 64 Pseudonymen deutschsprachiger Autoren kommt der Typ 6-mal vor. Die Zahl der Initialbildung in den übrigen Sprachen mag nicht ausreichend hoch für aussagekräftige Beobachtungen sein, soll aber dennoch hier zur Vollständigkeit genannt werden: unter den 81 französischen Autoren 3-mal und unter den spanischen Autoren 1-mal, also insgesamt bei $14 \%$ der historischen Pseudonyme (vgl. Tabelle 5). 


\begin{tabular}{|l|c|c|c|c|c|c|}
\hline & gesamt & engl. & dt. & frz. & it. & sp. \\
\hline \multirow{2}{*}{ Wikipedia } & 7 von $163=$ & 1 von $43=$ & 2 von $51=$ & 2 von $21=$ & 1 von $29=$ & 1 von $19=$ \\
& $4 \%$ & $2 \%$ & $4 \%$ & $10 \%$ & $3 \%$ & $5 \%$ \\
\hline \multirow{2}{*}{ historisch } & 85 von $607=$ & 75 von $438=$ & 6 von $64=$ & 3 von $81=$ & 0 von $10=$ & 1 von $14=$ \\
& $14 \%$ & $17 \%$ & $9 \%$ & $4 \%$ & $0 \%$ & $7 \%$ \\
\hline
\end{tabular}

Tabelle 5

Im historischen Vergleichskorpus absent sind Fälle von Deakronymisierung: Cépey $(<C$. P.), Aka $(<A K)<$ André Karwath, Efegé $(<F G)$.

\subsubsection{Anagrammatisierung}

Anagrammatisierung ist die Umstellung von Buchstaben. Zur Anagrammatisierung gibt es kein erkennbares Beispiel in unserem Wikipedia-Korpus. Unter den historischen Pseudonymen tauchen 12 Fälle auf (z. B. Ramon de Arriala für Mariano de Larra), davon weisen 5 Namen einfach die inverse Schreibung des Realnamens auf (z. B. Telba für William Ablett und Mit Yenda für Tim Adney).

\subsubsection{Buchstabenspiel bzw. Graphemspiel}

Unter Buchstabenspiel bzw. Graphemspiel sei der Austausch von Buchstaben bzw. Graphemen durch andere Buchstaben bzw. Grapheme verstanden. Buchstabenspiele sind selten unter den Wikipedia-Namen ebenso wie unter den historischen Pseudonymen. Beispielsweise schreibt sich der spanische Wikipedia-Autor Christian im Benutzernamen Krysthyan.

Eine für Wikipedia-Nicknames besondere Form des Graphemspiels kommt in den klassischen Pseudonymen nicht vor, nämlich der Ersatz von lateinischen Buchstaben durch ähnlich aussehende Zahlen oder Sonderzeichen. In der Internetwelt bezeichnet man dies als Leetspeak oder Leet, auch <1337> geschrieben. Das Wort kommt von élitespeak - also 'Codierung für gut eingeweihte Internet-Nutzer'. Darunter fallen in unserem Korpus die englischen Wikipedianer J04n und N4nojohn, der deutsche Wikipedianer CHR!S, der französische Wikipedianer MOtty und der spanische Wikipedianer Serg!o.

Überhaupt ist der Gebrauch von Zahlen ein Typ der Pseudonymenbildung, der bei den klassischen Pseudonymen nicht vorkommt. Darauf wird später noch einmal eingegangen.

\subsubsection{Kontamination/Blending}

Nicht genannt in den Terminologien von Bormann und Room ist der Typ Kontamination/ Blending/Kreuzung, der im Korpus der italienischen Wikipedia-Nicknames in Nicosmos, von Nico-Cosmos, steckt.

\subsubsection{Zwischenbilanz}

Die bislang genannten klassischen Typen sind auch in der Wikipedia möglich. Auffällig ist im historischen Vergleich die geringe Verwendung von Initialen unter den Wikipedia-Nicknames. Im sprachkontrastiven Vergleich fällt der hohe Gebrauch von Clippings bei den spanischen Wikipedia-Pseudonymen auf.

\subsection{Annahme des Namens einer anderen Person (heteronymische Pseudonyme)}

Wie der Name Sinatra aus dem Titel dieses Beitrags verrät, gibt es so genannte Heteronyme auch unter den Wikipedia-Nicknames. Das können lebende Personen oder verstorbene Personen sein (z. B. beim französischen Wikipedianer Caton oder bei den Wikipedianern mit 
Namen Sinatra). Oder es kann sich auch um mythologische oder literarische Personen handeln, z. B. HansCastorp (Figur aus Thomas Manns Zauberberg) oder der italienische Pap3rinik - wieder mit Leetspeak (entspricht im Deutschen Superduck oder Phantomias, dem Alter Ego von Donald Duck beziehungsweise - in Italien - Donaldo Papero). Die Häufigkeit solcher Fälle lässt sich nicht mit großer Sicherheit bestimmen - dies gilt für das WikipediaKorpus genauso wenig wie für das historische Korpus. Kombinationen aus Vor- und Nachnamen können wegen des Wohlklangs gewählt worden sein; Vor- und Nachname können von verschiedenen Personen stammen; etc.

\subsection{Sachliche Anlehnung an Namensträger (non-anthroponymische Pseudonyme)}

Die Umschreibung "sachliche Anlehnung an Namensträger" geht auf Bormann (1901: 23) zurück und soll ausdrücken, dass diese Art Pseudonym nicht auf einen Personennamen, ein Anthroponym gründet. Daher wird hier der Ausdruck non-anthroponymisches Pseudonym vorgeschlagen. Dieser Typ wird bei Bormann (1901) nicht weiter unterschieden, während Room (1981: 33, 40-42) noch zwischen (1) Pseudonymen mit Ortsangaben und (2) Pseudonymen, die keine Ortsangaben beinhalten, unterscheidet. Sie seien hier lokative Pseudonyme und nicht-lokative Pseudonyme genannt. Dabei können beide Typen natürlich wieder miteinander gekreuzt sein.

\subsubsection{Lokative Pseudonyme}

Unter die lokativen Pseudonyme fallen Namen, die auf den ehemaligen oder derzeitigen Wohnort zurückgehen. Es lassen sich auch verschlüsselte Ortsnamen hierher stellen, wie etwa Wahrerwattwurm, der dem Leser verrät:

Ich lebe im Watt der Unterelbe - genauer: in 'Hamburgs schöner Schwester' [= HamburgAltona].

Der Inhalt 'Einwohner von Hamburg-Altona' wird hier also metaphorisch und metonymisch verschlüsselt. Der Ortsname kann auch einen erträumten Ort beinhalten, wie beim englischen Wikipedianer Mermaid from the Baltic Sea, auf dessen Benutzerseite es heißt:

"Mermaid from the Baltic Sea is not actually a mermaid, and not actually from the Baltic Sea" ['Mermaid from the Baltic Sea ist nicht wirklich eine Meerjungfrau und nicht wirklich von der Ostsee'].

Aus dem historischen Korpus seien genannt: Aberdeen, A Scot und Scoto-Britannicus, die alle für James Anderson stehen.

\subsubsection{Non-lokative Pseudonyme (Einzelwörter oder Wortgruppen)}

Bei der hier vorgestellten Kategorie unterscheidet Room (1981: 34, 43-47, 60-63) zwischen (1) Wortgruppen, die wie Vorname und Nachname aussehen, (2) Rufname plus Appellativum bzw. Modifikator, (3) deskriptiven Titeln. Dabei tauchen die ersten beiden Untertypen sowohl im Wikipedia-Korpus als auch im historischen Korpus sehr selten auf.

\section{(1) Wortgruppen, deren erster Teil wie ein Rufname aussieht}

Das bekannteste Beispiel für diesen Typ ist Samuel Clemens' Künstlername Mark Twain, der auf eine Wendung aus der Schifffahrtssprache zurückgeht und 'Markiere zwei [Faden]' bedeutet. Aus unserem Wikipedia-Korpus lässt sich höchstens LilHelpa und Lilplayboii (bei Annahme eines vermeintlichen Vornamen $\mathrm{Lil}$ ) hierherstellen; auch im historischen Korpus ist dieser Typ nur 4-mal zu finden. 


\section{(2) Rufname plus Appellativum bzw. Modifikator}

In unserem Beispiel taucht diese Kombination mit dem Rufnamen als erstem Bestandteil im Korpus möglicherweise 2-mal auf, beim englischen Tomeasy und beim deutschen Jean-Pierre Hecht. Im historischen Korpus findet sich dies dagegen 13-mal, d. h. bei $3 \%$.

Ein Untertyp mit dem Rufnamen an zweiter Stelle liegt vor bei den englischen Wikipedianern Phantomsteve und Furrykef, letzterer heißt eigentlich Kef Schecter. Insgesamt kommt dies 3mal unter den englischen und 2-mal unter den deutschen Wikipedia-Namen (Lustiger seth, PaterMcFly) und 1-mal unter den französischen Wikipedia-Namen (ColonelSmith) unseres Korpus vor. Ähnlich liegen die Zahlen für das historische Korpus.

\section{(3) Deskriptive Titel}

Ein Beispiel für einen deskriptiven Titel - dies ist Rooms (1981: 3 et passim) Terminus - ist der deutsche Wikipedia-Nickname Baumeister, der explizit auf den Beruf des Namensträgers zurückgeht. Ferner können Vorlieben sowie echte oder gewünschte Eigenschaften zu Grunde liegen, etwa beim deutschen Herr-Schlauschlau, beim spanischen Ultrapedante, beim französischen Croquant (eigentlich ein Gericht aus karamellisiertem Zucker und Mandeln). Bei Ultrapedante zeigt sich auch, dass manche in ihrem Benutzernamen die Aufgabe ausdrücken, die sie sich für ihre Wikipedia-Arbeit gestellt haben: Korrektur von Verstößen gegen Interpunktionsregeln und Orthographie. Einer ähnlichen Aufgabe scheint in der deutschen Wikipedia der Autor Augiasstallputzer nachzugehen. Allerdings muss ein solcher Titel nicht der Wahrheit entsprechen. Wenn Voltaire bisweilen als Archevêque de Cantorbury 'Erzbischof von Canterbury' veröffentlicht, liegt dieser Bezeichnung kein Wahrheitsgehalt zu Grunde. Der selbstgewählte Name kommuniziert eben nicht notwendigerweise etwas über den Träger im Sinne der Grice'schen Maxime der Qualität. Daher bietet sich eher die Etikettierung "echt deskriptive Titel und pseudo-deskriptive Titel" an. Ein besonderer Typ von deskriptiven Titeln, der in Studien zu den klassischen Pseudonymen nicht auftaucht, ist der Gebrauch von Morphemen mit nichtgegenständlicher/nichtkonkreter, sondern abstrakter Bedeutung. Man kann dies als abstrakt-deskriptive Titel bezeichnen. Den Namen Elchjagd etwa hat sich eine Person gewählt, in deren Leben Elchjagd in irgendeiner Form eine Rolle spielt. In der Tat heißt es auf der entsprechenden Benutzerseite:

Die Elchjagd findet jeden Herbst in Schweden statt und ist von großer gesellschaftlicher

Bedeutung. [....] Ursprünglich bin ich aus Berlin, lebe jedoch seit 35 Jahren in Schweden.

Im Fall Elchjagd liegt folglich gleich eine poly-metonymische Motivierung vor. Wenngleich dieser Typ in den Studien zu den klassischen Pseudonymen nicht genannt wird, so ist er doch nachweisbar, beispielsweise bei dem schottischen pädagogischen Publizisten Promotion in the Church, der eigentlich William Angus heißt.

Die echt deskriptiven, pseudo-deskriptiven und abstrakt-deskriptiven Pseudonyme können sein:

(a) lexikalisierte, d. h. kodifizierte Wörter

(b) Adhoc-Bildungen

(c) Phrasen

(d) (Teil-)Sätze.

Die Verwendung von Phrasen ist besonders in der englischen Wikipedia auffällig. Dort tauchen sie im Korpus 26-mal auf, zu weniger als einem Viertel davon in den übrigen Wikipedia-Versionen. Beispiele sind: Mermaid from the Baltic Sea, LilHelpa, Interestedinfairness, Esprit fugace, Weiter Himmel. Bei Room ungenannt und in der Tat auch rar ist ein Untertyp 4.3.2.3d "Clause", der 1-mal unter den englischen Wikipedia-Namen auftaucht: 
Trust Is All You Need. In der Verwendung dieses Typs 4.3.2.3d gibt es deutliche Unterschiede zwischen den untersuchten Sprachen, wie Tabelle 6 zeigt.

\begin{tabular}{|l|c|c|c|c|c|}
\hline & engl. & fr. & it. & sp. & dt. \\
\hline $4.3 .2 .3 \mathrm{a}$ & 12 von $193=$ & 5 von $139=$ & 0 von $95=$ & 3 von $82=$ & 11 von $185=$ \\
& $6 \%$ & $4 \%$ & $0 \%$ & $4 \%$ & $7 \%$ \\
\hline $4.3 .2 .3 \mathrm{~b}$ & 7 von $193=$ & 4 von $139=$ & 3 von $95=$ & 1 von $82=$ & 9 von $185=$ \\
& $4 \%$ & $3 \%$ & $3 \%$ & $1 \%$ & $6 \%$ \\
\hline $4.3 .2 .3 \mathrm{c}$ & 26 von $193=$ & 4 von $139=$ & 1 von $95=$ & 0 von $82=$ & 6 von $185=$ \\
& $13 \%$ & $3 \%$ & $1 \%$ & $0 \%$ & $3 \%$ \\
\hline \multirow{2}{*}{$4.3 .2 .3 \mathrm{~d}$} & 1 von $193=$ & 0 von $139=$ & 0 von $95=$ & 0 von $82=$ & 0 von $185=$ \\
& $1 \%$ & $0 \%$ & $0 \%$ & $0 \%$ & $0 \%$ \\
\hline \multirow{2}{*}{ Summe } & 46 von $193=$ & 13 von $139=$ & 4 von $95=$ & 4 von $82=$ & 26 von $185=$ \\
& $24 \%$ & $9 \%$ & $4 \%$ & $5 \%$ & $16 \%$ \\
\hline
\end{tabular}

Tabelle 6

Im historischen Vergleich ist der Typ 4.3.2.3c im Englischen sogar noch stärker - er ist unter den historischen Pseudonymen unseres Korpus 184-mal vertreten, d. h. in $42 \%$ der Fälle, und damit der häufigste Typ; Typ 4.3.2.3b taucht im historischen Korpus nie auf; Typ 4.3.2.3a taucht 7-mal auf (d. h. zu $2 \%$ ). Typ 4.3.2.3 ist also unter den englischen historischen Pseudonymen insgesamt zu $44 \%$ vertreten.

\subsubsection{Weitere Aspekte}

Betrachten wir die bisherigen Typen von Pseudonymen, so lassen sich also ähnlich wie bei Familiennamen Herkunftsnamen, Rufnamen und Eigenschaftsnamen finden. Dabei können auch Kombinationen all dieser Typen und Untertypen vorliegen, z. B. bei den Wikipedianern Berlin-Jurist (Herkunft + Beruf) und Doric loon (Herkunft = 'bäurisch/schottisch' + Eigenschaft $=$ schottisch für 'Kerl').

Der letzte Fall, Doric loon, führt noch zu einer weiteren Anmerkung. Nicht-standardsprachliche Formen werden in der Literatur zu den herkömmlichen Pseudonymen nicht gelistet, aber in der Wikipedia tauchen sie auf. Unter den italienischen Wikipedianern ist bereits Vituzzu genannt worden, ein süditalienischer Diminutiv zu Vito, dem Realvornamen des Benutzers. Unter den englischen Wiki-Nicknames findet man Doric Loon (engl. doric 'bäurisch = schottisch', < '(bäuerisch-)dorisch (als Gegensatz zu städtisch-athenisch)', schott. loon 'Kerl'), LilHelpa, Biglovinb. Zum Teil liegt Augendialekt, "eye dialect", vor: Helpa wird im Britischen nicht anders ausgesprochen als Helper, die Aussprache lovin' beispielsweise ist auch in der gehobenen Umgangssprache. In unserem historischen Korpus ist allenfalls Honoré de Balzacs Anagramm (Lord) R'Hoone ein Beispiel für Augendialekt (für Rhône).

Unter den Pseudonymen des Typs 1 wurde bereits auf die Verwendung von fremdsprachlichen Elementen eingegangen. Auch die Verwendung von anderssprachigen Wörtern und Phrasen ist zu finden. Bei den Nicknames mit fremden Elementen ergibt sich ein deutliches Gefälle von den englischen Nicknames über die spanischen, französischen und italienischen Nicknames hin zu den deutschen Nicknames (vgl. Tabelle 7). 


\begin{tabular}{|c|c|c|c|c|c|}
\hline gesamt & engl. & dt. & fr. & it. & sp. \\
\hline 76 von $694=$ & 8 von $140=$ & 32 von $185=$ & 20 von $139=$ & 13 von $95=$ & 6 von $82=$ \\
$11 \%$ & $4 \%$ & $17 \%$ & $14 \%$ & $14 \%$ & $7 \%$ \\
\hline
\end{tabular}

Tabelle 7

Tabelle 8 zeigt, welche Sprachen als Gebersprachen für die fremden Elementen in den einzelnen Sprachen dienten.

\begin{tabular}{|l|c|c|c|c|c|}
\hline & engl. & dt. & fr. & it. & sp. \\
\hline Ashanti & 1 & & & & \\
\hline Deutsch & & & 1 & 1 & \\
\hline Englisch & & 20 & 11 & 8 & 2 \\
\hline Französisch & 1 & 1 & & & \\
\hline Griechisch & & 1 & 1 & & \\
\hline Hindi & 1 & & & & \\
\hline Italienisch & & 1 & & & \\
\hline Japanisch & 2 & 2 & & & \\
\hline Lateinisch & 2 & 3 & 4 & & 1 \\
\hline Russisch & & 1 & & & \\
\hline
\end{tabular}

Tabelle 8

Demnach steht englisches Fremdgut in den nicht-englischen Wikipedias an erster Stelle, besonders deutlich unter den deutschen Nicknames (danach unter den italienischen, spanischen und französischen Nicknames). Tabelle 9 zeigt den Anteil der englischen Elemente unter den fremdsprachlichen Elementen in den verschiedenen Nickname-Gruppen.

\begin{tabular}{|c|c|c|c|}
\hline dt. & fr. & it. & sp. \\
\hline 20 von $32=63 \%$ & 11 von $20=55 \%$ & 8 von $13=62 \%$ & 2 von $6=33 \%$ \\
\hline
\end{tabular}

\section{Tabelle 9}

Tabelle 10 zeigt den Anteil an englischen Elementen gemessen an der Gesamtzahl der berücksichtigten Nicknames.

\begin{tabular}{|c|c|c|c|}
\hline dt. & fr. & it. & sp. \\
\hline 20 von $185=11 \%$ & 11 von $139=8 \%$ & 8 von $95=8 \%$ & 2 von $82=2 \%$ \\
\hline
\end{tabular}

Tabelle 10

Diese Reihung ähnelt sehr den Ergebnissen von Untersuchungen zum Anglizismen-Anteil bei Appellativa. So erbrachte eine Analyse von 1.941 Lemmata aus Görlachs (2001) Dictionary of European Anglicisms und eine Analyse von 599 Einzelbedeutungen in Görlachs Wörterbuch (Grzega 2006: 107f.) die in Tabelle 11 gezeigte Verteilung. 


\begin{tabular}{|l|c|c|c|c|}
\hline Verteilung von Anglizismen & dt. & fr. & it. & sp. \\
\hline Korpus bei Görlach 2001 & $70.9 \%$ & $52.7 \%$ & $53.6 \%$ & $46.7 \%$ \\
\hline Korpus bei Grzega 2006 & $57.4 \%$ & $47.6 \%$ & $48.2 \%$ & $46.2 \%$ \\
\hline
\end{tabular}

Tabelle 11

Freilich sind die Methoden zur Gewinnung dieser Zahlen nicht gänzlich vergleichbar, dennoch lässt sich Folgendes bemerken: Gemessen an den Studien zu den nicht-onymischen Anglizismen wäre bei den onymischen Wikipedia-Korpora zum Französischen und Italienischen ein größerer Abstand seitens des Deutschen und ein geringerer Abstand seitens des Spanischen zu erwarten gewessen.

Unter den 549 historischen Pseudonymen tauchen in $12 \%$ der Fälle fremdsprachliche Elemente auf. Das entspricht dem Anteil fremdsprachlicher Elemente unter den WikipediaPseudonymen. Aber die Prominenz der Gebersprachen hat sich geändert. Besonders auffällig ist der Rückgang der lateinischen Elemente: unter den 67 historischen Pseudonymen mit Fremdgut waren es 35-mal lateinische Elemente, d. h. in $52 \%$ der Fälle. Unter den modernen Wikipedia-Nicknames ist unter den 76 Pseudonymen mit Fremdgut nur 7-mal Lateinisches, d. h. in nur $9 \%$ (vgl. Tabelle 12).

\begin{tabular}{|l|l|l|}
\hline & historisch (alle Sprachen) & WP (alle Sprachen) \\
\hline Fremdes & 67 von $549=12 \%$ & 76 von $694=11 \%$ \\
\hline Lat. & 35 von $67=52 \%$ & 7 von $76=9 \%$ \\
\hline
\end{tabular}

Tabelle 12

Dieser Rückgang des Lateinischen könnte dreierlei Gründe haben: (1) Die Bedeutung des Lateinischen als europäischer bzw. westlicher Verkehrssprache ist fast völlig verloren gegangen. (2) Die Bedeutung des Lateinischen als europäischer bzw. westlicher Schulsprache ist stark reduziert. (3) Hinter den Wikipedianern können sich auch Benutzer verbergen, die nicht aus dem lateinisch-geprägten europäischen oder westlichen Raum kommen.

In den 438 historischen englischen Pseudonymen tauchen in 57 fremde Elemente auf, was dem durchschnittlichen Anteil fremdsprachlichen Materials unter Berücksichtigung aller historischen Pseudonyme entspricht. Dagegen zeigt sich bei den englischen WikipediaPseudonymen ein auffälliger geringerer Anteil an fremden Elementen (vgl. Tabelle 13).

\begin{tabular}{|l|l|l|}
\hline & gesamt & englisch \\
\hline Fremdelemente, historisch & 67 von $549=12 \%$ & 57 von $438=13 \%$ \\
\hline Fremdelemente, Wikipedia & 76 von $694=11 \%$ & 8 von $140=4 \%$ \\
\hline
\end{tabular}

Tabelle 13

Die geringe Bedeutung des fremdsprachlichen Elements in den englischsprachigen Wikipedia-Pseudonymen ist zunächst erstaunlich angesichts der Tatsache, dass die Gruppe der englischsprachigen Wikipedianer die internationalste sein dürfte. Möglicherweise möchten aber gerade nicht-englische Muttersprachler ihre Englisch-Kompetenz beweisen und wählen daher einen für das Internet typischen englischen Namen. 


\subsection{Zeichen und Symbole}

Der Typ "Pseudonyme aus Zeichen und Symbolen, die nicht (lateinische) Buchstaben sind" wird bei Room (1981: 47) nur sehr kurz behandelt. Room erwähnt (1981: 47), dass vor allem der Asterisk und der Gedankenstrich eingesetzt wurden. So verwendeten George Orwell und Samuel Clemens alias Mark Twain mitunter einen einfachen Asterisken. Ähnliches erfährt man bereits bei Cushing (1886). Dort findet man unter anderem folgende Symbole (Cushing ebd.: 1):

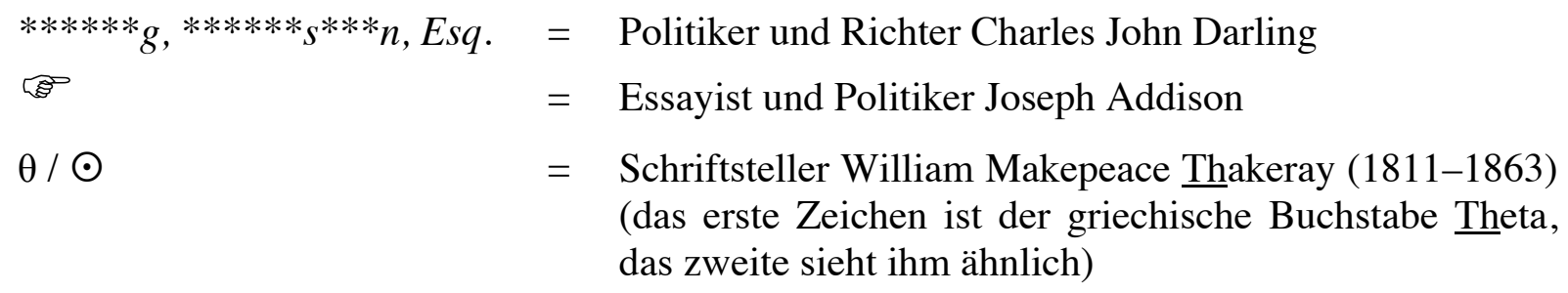

die Musiknoten E, H, C = Eliza Brown Chase (Autorin des späten 19. Jh.; im Eng lischen blieb der ursprüngliche Name der Note $H$, nämlich $\mathrm{B}$, erhalten)

Insgesamt scheint dieser Typ eher selten zu sein. In unserem historischen Korpus taucht dies 5-mal auf. Auch im Wikipedia-Korpus ist die Verwendung von Sonderzeichen verhältnismäßig selten, aber es gibt sie. Die Gruppe der Sonderzeichen bleibt jedoch auf das beschränkt, was mit dem Standard-Tastaturensatz, dem sog. ASCII (American Standard Code for Information Interchange) möglich ist. Also: Dollarzeichen und lateinische Buchstaben mit nationalen Diakritika ja, Musiknoten und griechische Buchstaben nein.

Umgekehrt war auch die bewusste Vermeidung von nationalen Sonderzeichen bei fünf deutschen Wikipedianern zu finden: bei Buncic, eigentlich Daniel Bunčić, bei JakobVoss, eigentlich Jakob Voß, und die Umlautauflösungen bei Woehlecke, eigentlich Matthias Wöhlecke, sowie bei BesondereUmstaende und Westfalenbaer, wie auch immer ihre Realnamen seien.

Zusätzlich zu Rooms Pseudonymentypen existiert in der Wikipedia ein Typ Zahlennamen der Gestalt $A B 123$. Freilich können auch in Realnamen Zahlwörter stecken, zumindest synchron gesehen. Beispiele wären Viereck (Übername für 'klotzig' oder Wohnstättenname oder Ortsname in Mecklenburg-Vorpommern), Dreyfuss (eigentlich Trevus 'jemand aus Trier'), Zweipfennig (Übername nach Zins-/Abgabepflicht), Einstein (Ortsname im Elsass: ein = 'allein, einzeln'; Stein = 'Fels'). Aber diese können weder allein deskriptiver Titel sein noch mit Ziffernsymbolen geschrieben werden. Bei den Wikipedia-Namen ist dies anders.

Ein Vergleich der verschiedenen Sprachversionen (vgl. Tabelle 14) ergibt versionsübergreifend keine großen Ausscherungen unter den Zahlennamen. In der deutschen Version ist der Typ aber im direkten Vergleich zur französischen Version deutlich häufiger. Der deutsche Prozentsatz liegt auch deutlich über und der französische deutlich unter dem durchschnittlichen Prozentsatz.

\begin{tabular}{|c|c|c|c|c|c|}
\hline gesamt & engl. & dt. & fr. & it. & sp. \\
\hline 114 von $694=$ & 28 von $193=$ & 45 von $185=$ & 12 von $139=$ & 18 von $95=$ & 11 von $82=$ \\
$16 \%$ & $15 \%$ & $24 \%$ & $9 \%$ & $19 \%$ & $13 \%$ \\
\hline
\end{tabular}

Tabelle 14

In einer Kombination aus einer Ziffernfolge plus einem Rufnamen oder einem deskriptiven Titel kann die Ziffernfolge unterschiedlich motiviert sein: 
(a) Alter (bei der Anmeldung), z. B. beim deutschen Benutzernamen Kolja21

(b) Geburtsjahr, z. B. beim italienischen Benutzernamen Giuse93

(c) Geburtsdatum, z. B. beim spanischen Benutzernamen David0811

(d) Sonstiges (Spiel), z. B. bei den deutschen Benutzernamen justyjusty123 und He3nry.

An diesem letzten Beispiel sehen wir, dass es manchmal schwierig ist, solche geschriebenen Pseudonyme zu sprechen. Schlecht zu verbalisieren sind auch die Nicknames ZZuuzz, Lmaltier, Dch, Ejrrjs und Andrsvoss. Dies würde im Schriftverkehr auch keine weitere Schwierigkeit bereiten, wären nicht auch die Diskussionsseiten der Wikipedia zwar - im Sinne von Koch/Oesterreicher (1985) - ein schriftliches Medium, aber doch von der Konzeptionalität eher "gesprochen". Daher lässt sich hier im Gegensatz zu den historischen Nicknames noch ein weiteres Phänomen beobachten. Es kann neben der Anrede mit WikipediaNickname, der Anrede mit Realnamen und - sehr häufig - einer fehlenden Anrede mit Namen in Diskussionen auf den Wikipedia-Seiten zu Kürzungen der Wikipedia-Benutzernamen kommen. Es kommt dann, wenn man so will, zu "Nicknicknames". Im Gegensatz zu den autonomen Internet-Nicknames sind die Nicknicknames wieder heteronom. Wie diese aussehen, soll im nächsten Abschnitt thematisiert werden.

\section{Korpusanalyse 2: Anmerkungen zu den (heteronymen) Nicknicknames}

Nicknicknames sind eine Form von Kosenamen. Der Terminus Kosename wird unterschiedlich weit verwendet: in einem weiteren Sinne wird dabei jede Form von inoffiziellem Eigennamen verstanden; in einem engeren Sinne (etwa bei Naumann 1995) werden darunter nur solche inoffiziellen Namen verstanden, die von den offiziellen abgeleitet sind. Nur der letzte Fall kann bei Nicknicknames vorliegen (außer man zählt auch die Anrede mit dem Realnamen, der nicht Basis der Nicknames ist, zu den Nicknicknames).

Naumann (1995) unterscheidet folgende Typen von Kosenamen, die von Eigennamen abgeleitet sind: (1) Kürzung von Bestandteilen (z. B. Johannes $>$ Jo, Johann, Hannes, Hans), (2) Suffigierung (z. B. Hans > Hansi), (3) Kontraktion (z. B. Bernhard > Bernd, Rosemarie > Romy), (4) Lallnamen (z. B. Josef (>Sepp) > Beppi). Dies könnte zunächst als Grundlage für unsere Einteilung dienen. Es fällt aber auf, dass von diesen Typen nur die Kürzung in unserem Korpus vorkommt. Dagegen tauchen im Korpus noch weitere Typen auf, die in einer Klassifikation ergänzt werden müssen.

Ein quantitativer Vergleich von Nicknickname-Typen ist wenig sinnvoll, denn die Möglichkeiten ergeben sich ja nur durch die Langformen der Nicknames. Es lässt sich aber sinnvoll eine quantitative Aussage über den Gebrauch von Nicknicknames allgemein in den verschiedenen Wikipedias treffen. So gibt es bei der in Tabelle 15 dargestellten Anzahl von Wikipedianern Nicknicknames in einer oder mehreren der obigen Formen.

\begin{tabular}{|c|c|c|c|c|c|}
\hline gesamt & engl. & dt. & fr. & it. & sp. \\
\hline 237 von $694=$ & 61 von $193=$ & 63 von $185=$ & 32 von $139=$ & 44 von $95=$ & 37 von $82=$ \\
$34 \%$ & $32 \%$ & $34 \%$ & $23 \%$ & $46 \%$ & $45 \%$ \\
\hline
\end{tabular}

Tabelle 15

Dabei sticht der hohe Anteil der Nicknicknames in der italienischen und spanischen Wikipedia ins Auge.

\subsection{Kürzung eines Bestandteils}

Unter der Rubrik Kürzung bzw. Tilgung eines Bestandteils ließen sich noch weitere Untertypen schaffen. Ein Untertyp besteht etwa in der Tilgung von suffix-artigen Ziffern, die man 
als Ziffernsuffixoide bezeichnen könnte und die determinierenden bzw. spezifizierenden Charakter haben. Die Wikipedianer Chrono1084 und Giuse93 beispielweise werden auf der Diskussionsseite nur noch mit Chrono und Giuse angesprochen. Es kann aber auch Tilgung eines Buchstabensuffixoids erfolgen: Besonders bei Kombinationen aus einem Rufnamen und einem weiteren Buchstabenbestandteil (Initial oder Nachname) wird letzterer gerne weggelassen: NeilN wird mit Neil (und Marco Bernardini mit Marco) angesprochen - egal, ob dies auch die gesicherten Realnamen sind oder nicht. Des Weiteren kann an einer Interpunktionsstelle (Bindestriche, Leerstellen und Punkte) getilgt werden: Socho-sama wird dann mit Socho, Nihil novi mit Nihil, Octave.H mit Octave und Marcol-it mit Marcol angesprochen.

Ein zweiter Untertyp besteht in der bloßen Tilgung der Nicht-Buchstaben: Die Franzosen PicSou und (:Julien:) werden dann als Picsou und Julien angesprochen.

Ein weiterer Untertyp ist die Tilgung eines morphematischen Bestandteils (Ellipse), bei der ein Name übrig bleibt. Korpus-Beispiele sind folgende: unter den englischen Users wird Phantomsteve zu Steve, unter den deutschen Benutzern wird Lustiger seth zu Seth, unter den französischen Utilisateurs wird Stanlekub zu Stan, unter den italienischen Utenti wird Fabiosbaraglia zu Fabio, unter den spanischen Usuarios wird Andreasmperu zu Andrea und Andreas - mit unklarer Morphemgrenze und damit unklarem Geschlecht (ist es eine Andrea $S$. M. aus Peru oder ein Andreas M. aus Peru?). Korpus-Beispiele für die Tilgung eines morphematischen Bestandteils (Ellipse), bei der ein Appellativum bzw. ein appellativisches Morphem übrig bleibt, sind folgende: unter den englischen Users wird Mermaid from the Baltic Sea zu Mermaid und CapitalR zu Capital (im letzten Fall bleibt also das Determinans, nicht das Determinatum übrig, als handelte es sich um eine Vorname-NachnameKombination), unter den französischen Utilisateurs wird Orthogaffe zu Ortho, unter den italienischen Utenti wird MoreThanMeetsTheEye zu MoreThan und More, unter den spanischen Usuários wird Efegé zu Efe, unter den deutschen Benutzern wird Wahrerwattwurm zu Wurm, PaterMcFly manchmal zu McFly, meistens zu Pater und Liberal Freemason zu Liberal (auch in diesem letzten Fall bleibt das Determinans und nicht das Determinatum übrig). Die Determinatum-Tilgung ist deutlich häufiger als die Determinans-Tilgung: erstere taucht 13-mal auf, letztere nur 2-mal. Offenbar werden hier komposite Formen wie ein Zusammensetzung aus Vorname und Familienname betrachtet. Da es in der Wikipedia-Gemeinde üblich ist, sich zu duzen, ist das Herausfiltern eines inoffiziellen Pseudo-Vornamens aus dem offiziellen Wikipedia-Benutzernamen ein nachvollziehbarer Vorgang.

Von der Ellipse trennen könnte man die Wortkürzung bzw. das Clipping als Tilgung eines nicht-morphematischen Bestandteils. Einige Beispiele aus dem Korpus für die Tilgung eines nicht-morphematischen Bestandteils (Clipping), bei der ein neues Morphem übrig bleibt, sind folgende: Snowded wird mit Snow und mit Snowy angesprochen, Marcol-it, nicht nur als Marcol, sondern auch als Marco. Beispiele für die Tilgung eines nicht-morphematischen Bestandteils (Clipping), bei der auch kein Morphem übrig bleibt, finden sich im Korpus der italienischen Wikipedianer-Pseudonymen: Revares wird zu Rev und Erlkoenig wird zu Erl (weder Erl noch koenig haben im Italienischen Morphemstatus), und der deutsche Lirum Larum wird zu Lirum.

\section{$5.2 \quad$ Suffigierung}

Im Korpus wären hierzu nur (in Kombination mit einer vorangehenden Kürzung) die Bildungen von Berliner aus Berlin-Jurist und von Monty aus Montgomery zu nennen. 


\subsection{Kontraktion}

Die Kontraktion stellt eigentlich eine besondere Form der Kürzung dar. Eine typische Kontraktion lässt sich im Korpus nicht finden.

\subsection{Lallformen}

Unter die Rubrik Lallformen würde die Anrede von Giuse93 als Peppe fallen (in Kombination mit Tilgung der Ziffernfolge). Hier wird einfach die übliche alltagssprachliche Lallform für Giuseppe herangezogen. Weitere Beispiele tauchen im Korpus nicht auf.

\subsection{Initialbildung}

Die Initialbildung ist ein Kosenamen-Typ, den man im Europa des 20. und 21. Jh. vor allem aus den USA kennt (John Ross Ewing $>$ J. R. Ewing) (vgl. auch Ashley 1995: 1752). Er taucht im Alltag der anderen untersuchten Sprachgemeinschaft bestenfalls selten auf (vgl. dazu auch die entsprechenden Artikel in Brendler/Brendler 2007). Unter den zusammengetragenen Nicknicknames finden sich dagegen doch einige Fälle, und zwar neben den englischen vor allem unter den deutschen (vgl. Tabelle 16): Tabletop wird zu TT, Esprit Fugace zu EF, Wahrerwattwurm zu Wwwurm und Www, Berlin-Jurist zu BJ, Liberal Freemason zu LF, Trust Is All You Need zu TIAYN.

\begin{tabular}{|c|c|c|c|c|c|}
\hline gesamt & engl. & dt. & fr. & it. & sp. \\
\hline 13 von $114=$ & 5 von $28=$ & 7 von $45=$ & 1 von $12=$ & 0 von $18=$ & 0 von $11=$ \\
$11 \%$ & $18 \%$ & $16 \%$ & $8 \%$ & $0 \%$ & $0 \%$ \\
\hline
\end{tabular}

Tabelle 16

\subsection{Revertierung von Leetspeak}

Die Revertierung von Leetspeak kommt in unserem Korpus nur in den nicht-englischen Wikipedia-Gemeinden vor: der französische MOtty wird zu Motty, der spanische Serg!o zu Sergio und der deutsche CHR!S zu Chris.

\subsection{Sonstige Nicknicknames}

Einige Bildungen lassen sich in keine der genannten Kategorien einordnen. Dabei können die letzten beiden Phänomene auch nicht mit Ökonomie erklärt werden.

(a) die Verwendung des realen Vornamens, wenn dieser auf der Benutzerseite bekannt gemacht wird (selbst wenn der Benutzername völlig davon abweichen sollte): diese Anredeform kommt sehr häufig vor

(b) ZZuuzz zu Zuz

(c) $O O O D D D$ zu $3 O 3 D$

(d) PoLuX124 zu Polux: die inneren Majuskeln werden durch Kleinbuchstaben ersetzt (hier in Kombination mit Tilgung der Ziffernreihe)

(e) Kolossus zu Colosse (in der französischen Wikipedia), PassePorte zu Passport (in der deutschen Wikipedia) und Phantomas zu Fantomas (in der italienischen Wikipedia), Krysthyan zu Cristian und Christian (in der spanischen Wikipedia): diese Bildungen können als Indigenisierungen betrachtet werden

(f) Gons zu Gonzalo: die mutmaßliche Koseform wird durch die mutmaßliche Langform ersetzt

(g) Andrsvoss zu Andreas (obwohl so nicht bekannt, es könnte auch eine Andrea S. oder ein André S. oder sonst ein Name dahinter stecken) 


\section{$6 \quad$ Zusammenfassung}

Im qualitativen historischen Vergleich erweisen sich als typisch moderne Phänomene

(1) Leetspeak, also der Ersatz von Buchstaben durch ähnlich aussehende Ziffern und andere Grapheme (dies kann der eingeschränkten Wahl von internationalen NichtBuchstaben-Zeichen geschuldet sein, die sich durch die internationale Tastatur ergeben);

(2) die Verwendung von nicht-standardsprachlichen Formen (dies kann als Symptom der modernen Verwischung der Gebrauchsbedingungen für Standard und Nonstandard in Europa und Amerika gewertet werden);

(3) Deakronymisierung;

(4) die Schöpfung von Kosenamen von Pseudonymen, hier "Nicknicknames" genannt (eine Ausnahme bilden Initial(en) oder der erste Teil einer Phrase, die auch historisch - unter anderem zur Platzersparnis verwendet werden konnten).

Dagegen fehlen unter den Wikipedia-Autoren Symbole, die nicht Teil des ASCII-Satzes sind und somit nicht bequem über die Standard-Tastatur eingegeben werden könnten. Im quantitativen historischen Vergleich sticht vor allem der Rückgang lateinischer Elemente ins Auge. Dies geht mit der geschwundenen Bedeutung des Lateinischen als Verkehrs- und Schulsprache einher.

Im synchronen Vergleich fallen die einzelnen Wikipedianer-Gemeinschaften unterschiedlich auf:

(1) Die englischsprachigen Wikipedianer heben sich ab durch den hohen Grad an Phrasen als Pseudonyme, wie überhaupt den hohen Grad an non-lokativen non-anthroponymischen Pseudonymen, durch den hohen Grad an Zahlennamen und den niedrigen Grad an fremden Elementen.

(2) Die französischsprachigen Wikipedianer heben sich ab durch den geringen Grad an Offenlegung der Benennungsmotive ihrer Pseudonyme und durch den geringsten Grad an Zahlennamen.

(3) Die italienischsprachigen Wikipedianer heben sich ab durch den hohen Grad an Offenlegung der Benennungsmotive ihrer Pseudonyme, durch den geringen Grad an non-lokativen non-anthroponymischen Pseudonymen und durch den hohen Grad an Nicknicknames.

(4) Die spanischsprachigen Wikipedianer heben sich ab durch den geringen Grad an nonlokativen non-anthroponymischen Pseudonymen, durch den niedrigsten Anteil an fremden Elementen in ihren Nicknames, durch den hohen Grad an Nicknicknames und durch den höchsten Grad von Clippings.

(5) Die deutschsprachigen Wikipedianer heben sich ab durch den höchsten Anteil an fremden Elementen in ihren Nicknames.

Es sollen zumindest einige Erklärungsversuche für diese Merkmale angeführt werden.

- Möglicherweise lässt sich die geringe Zahl an Zahlen-Pseudonymen unter den französischsprachigen Wikipedianern, die vielleicht zum Großteil Franzosen sind, damit erklären, dass in Frankreich in förmlichen Kontexten sehr viel Wert auf rhetorische Finesse gelegt wird (Galtung (1983) nennt einen auf Ästhetik ausgerichteten, rhetorisch-stilistisch fokussierten Stil demzufolge auch "gallischen Stil") und dass Zahlen hier zu banal wirken könnten.

- Die Beliebtheit von meist kürzenden Nicknicknames in der italienischen Wikipedia mag die Beliebtheit von meist kürzenden Kosenamen im Realleben widerspiegeln (vgl. dazu Berardi 2007: 331f.). 
- Der hohe Grad an Clippings unter den spanischen Wikipedianern mag aus der Diskrepanz entstehen, dass in der Wikipedia, wie im Internet allgemein, einerseits nach Kürze gestrebt wird und spanische (und lateinamerikanische) Namen prinzipiell mehrteilig sind.

- Der hohe Fremdanteil in deutschen Wikipedia-Nicknames mag die generell nachweisbare Offenheit für Lehngut fortführen.

\section{Einschränkungen}

Die Studie kann natürlich nur ein erster Schritt der Beschreibung und Erklärung von Wikipedia-Pseudonymen sein. Bei den Vergleichen ist zu berücksichtigen, dass die Realnamen hinter den historischen Pseudonymen immer bekannt sind, dass aber bei den WikipediaPseudonymen nur für einen Teil die Realnamen bekannt sind; umgekehrt ist das Benennungsmotiv für ein Wikipedia-Pseudonym oftmals klarer als für ein Pseudonym der Autoren im historischen Korpus. Kultur- bzw. sprachbezogene Erklärungen sind für die englischen Wikipedia-Nicknames sehr vorsichtig zu formulieren, da gerade die englische Wikipedia keine Wikipedia-Version ist, in der sich vor allem Autoren mit englischer Sprache tummeln, sondern eine hohe Anzahl an Nicht-Muttersprachlern an der Erstellung mitwirkt (andererseits haben aber auch Autoren unseres historischen Korpus nicht nur in ihrer Muttersprache geschrieben).

\section{Perspektiven}

Zum Schluss stellt sich die Frage: Was sind weitere Perspektiven im Zusammenhang mit der Internet-Nickname-Forschung (wozu meist eine größere Datenbasis notwendig sein wird)? Einige weitere Fragen, denen man nachgehen kann, sind folgende:

- Ist die Bildung von Benutzernamen von den Interessen der Wikipedianer abhängig? (Einbezug von Lemmata unterschiedlicher Sachgebiete)

- Hängt die Quantität von Clippings unter den hispanophonen Wikipedianern mit der Mehrgliedrigkeit der Namen zusammen? Wie häufig ist dieser Typ in anderen Sprachräumen mit mehrgliederigen Namen (z. B. Russisch)?

- Geht die Quantität von englischsprachigen Elementen in einer Sprachversion mit dem Anteil von nicht-onymischen Anglizismen in der Allgemeinsprache einher?

- Sind spezifische Leetspeak-Phänomene sprachenabhängig? (z. B. In welcher Sprachversion steht die 4 häufiger für $h$, wo häufiger für $A$ ?)

- Gibt es Regelmäßigkeiten oder Tendenzen bei der Bildung von Nicknicknames? Ist etwa die Quantität von Determinatumstilgungen bei der Bildung von Nicknicknames sprachenspezifisch?

Die Nickname-Forschung könnte so nicht nur interessante sprachsystemische, sondern auch interessante sprachpragmatische und psycholinguistische Aspekte zu Tage fördern.

\section{Literatur}

Ashley, Leonard R. N. (1995): "Nicknames and Sobriquets". In: Eichler, Ernst et al. (eds.) (1995): Namenforschung. Ein internationales Handbuch zur Onomastik/Names Studies. An International Handbook of Onomastics/Les noms propres. Manuel international d'onomastique. 2 vol. Berlin/New York: 1750-1756.

Berardi, Rocco (2007): "Das italienische Personennamensystem". In: Brendler, Andrea/ Brendler, Sivio (eds.) (2007): Europäische Personennamensysteme. Ein Handbuch von Abasisch bis Zentralladinisch. Hamburg: 322-338.

Brendler, Andrea/Brendler, Sivio (eds.) (2007): Europäische Personennamensysteme. Ein Handbuch von Abasisch bis Zentralladinisch. Hamburg. 
Bormann, Edwin (1901): Die Kunst des Pseudonyms. 12 literarhistorisch-bibliographische Essays. Leipzig.

Cushing, William (1886): Initials and Pseudonyms. A Dictionary of Literary Disguises. London.

Dauzat, Albert (1942): Les noms de personnes, origine et évolution. Prénoms, noms de famille, surnoms, pseudonymes. Paris.

Eichler, Ernst et al. (eds.) (1995): Namenforschung. Ein internationales Handbuch zur Onomastik/Names Studies. An International Handbook of Onomastics/Les noms propres. Manuel international d'onomastique. 2 vol. Berlin/New York.

Galtung, Johan (1983): "Struktur, Kultur und intellektueller Stil. Ein vergleichender Essay über sachsonische, teutonische, gallische und nipponische Wissenschaft". Leviathan 2: 303-338.

Görlach, Manfred (2001): Dictionary of European Anglicisms. A Usage Dictionary of Anglicisms in Sixteen European Languages. Oxford.

Grzega, Joachim (2006): EuroLinguistischer Parcours. Kernwissen zur europäischen Sprachkultur. Frankfurt am Main.

Koch, Peter/Oesterreicher, Wulf (1985): "Sprache der Nähe - Sprache der Distanz. Mündlichkeit und Schriftlichkeit im Spannungsfeld von Sprachtheorie und Sprachgeschichte". Romanistisches Jahrbuch 36: 15-43.

Naumann, Horst (1995): "Kosenamen". In: Eichler, Ernst et al. (eds.) (1995): Namenforschung. Ein internationales Handbuch zur Onomastik/Names Studies. An International Handbook of Onomastics/Les noms propres. Manuel international d'onomastique. 2 vol. Berlin/New York: 1757-1761.

Ratier, Emmanuel (1993-1994): Encyclopédie des pseudonymes. 2 vol. Paris.

Room, Adrian (1981): Naming Names. Stories of Pseudonyms and Name Changes with a Who's Who. London. 